\title{
Transgender-Related Insurance Denials as Barriers to Transgender Healthcare: Differences in Experience by Insurance Type
}

\author{
Matthew Bakko, MSW, MA ${ }^{\top}$ (]) and Shanna K. Kattari, PhD, MEd ${ }^{2}$ \\ 'School of Social Work, Department of Sociology, University of Michigan, Ann Arbor, MI, USA; '2School of Social Work, Department of Women's \\ Studies, University of Michigan, Ann Arbor, MI, USA.
}

\begin{abstract}
BACKGROUND: Insurance-based denials are common barriers for transgender and non-binary individuals in accessing medically necessary gender-affirming care. Little is known about how experiences of transgenderrelated insurance denials may vary by insurance type.

OBJECTIVE: This study investigates the association between transgender and non-binary individuals' experiences of different forms of transgender-related insurance denials and insurance type.
\end{abstract}

DESIGN AND PARTICIPANTS: The 2015 United States Transgender Survey was conducted by the National Center for Transgender Equality to ascertain US transgender and non-binary experiences across multiple life experiences, including individual health status, health services access and utilization, and experiences with denials.

MAIN MEASURES: Multivariate logistic regressions were conducted, and adjusted risk ratios were calculated, to analyze the likelihood of experiencing eight different forms of denials by insurance type, including private, Medicare, Medicaid, and military-related, and having multiple types of insurance coverage.

KEY RESULTS: Models revealed significant relationships between transgender-related insurance denials and insurance type for 11,320 transgender and non-binary adults. Compared with those with private insurance, Medicaid coverage was associated with an increased likelihood of experiencing denials for hormone therapy (adjusted risk ratio $(\mathrm{ARR})=1.22 ; \mathrm{CI}=1.05-1.42 ; p=0.02$ ); having no in-network surgery providers was associated with Medicare (ARR $=1.84 ; \mathrm{CI}=1.29-2.62 ; p=0.009$ ) or Medicaid $(\mathrm{ARR}=1.54 ; \mathrm{CI}=1.20-1.98 ; p=0.003)$; and militarybased insurance was associated with transition-related surgery denials $(\mathrm{ARR}=1.53 ; \mathrm{CI}=1.36-1.72 ; p<0.001)$.

CONCLUSIONS: Researchers and practitioners must consider the link between type of insurance coverage and experiences with different forms of transgenderrelated insurance denial. These results provide continuing support for broad non-discrimination policy efforts, but also direct our attention to targeted insurance policy interventions by form of denial, which can promote equitable access for transgender and non-binary people across all healthcare needs.

A version of this research was presented at the Annual Conference of the Society for Social Work and Research, January 18, 2020.

Received July 23, 2019

Revised December 25, 2019

Accepted February 5, 2020

Published online March 3, 2020
KEY WORDS: access to care; health insurance; denials; discrimination; transgender.

J Gen Intern Med 35(6):1693-1700

DOI: $10.1007 / \mathrm{s} 11606-020-05724-2$

(C) Society of General Internal Medicine 2020

\section{INTRODUCTION}

Transgender and non-binary (TNB) individuals have a variety of health needs that require accessible, affordable, and quality healthcare. For those seeking medical transition, this can include gender-affirming medical care such as hormone therapy, surgery, and support services like counseling. ${ }^{1-5}$ Genderaffirming medical care is the provision of transition-related medical services that supports a transgender person's own gender identity. It is considered medically necessary by the World Professional Association of Transgender Health (WPATH) and has been linked to the overall well-being of transgender individuals. ${ }^{4-7}$ It is also cost-effective when compared with the high cost of physical and mental healthcare that may result from being unable to access needed services. ${ }^{4,5,8} \mathrm{~A}$ plethora of research exists demonstrating that TNB individuals experience high rates of physical and mental health issues, including depression, anxiety, self-harm, suicidal ideation, and disability, ${ }^{3,9-16}$ which may result from lack of healthcare access, as well as stigma and transphobia. According to the 2015 United States Transgender Survey (USTS), the largest survey of the TNB population to date, $39 \%$ of respondents reported severe psychological distress, in comparison with $5 \%$ of the general population. ${ }^{12}$

However, the USTS also shows that $23 \%$ of respondents avoided seeking healthcare due to fear of mistreatment within the past year. ${ }^{12}$ Both negative health outcomes and delays in seeking care among TNB people can be attributable to perceived and direct experiences of mistreatment, stigma, and discrimination. ${ }^{17-20}$ Research shows that TNB experiences with healthcare discrimination are pervasive and include providers refusing to offer gender-affirming medical care, asking unnecessary questions about gender unrelated to the purpose of the healthcare visit, and lacking knowledge of trans-related health issues. ${ }^{12,21-23}$ Furthermore, TNB experiences with healthcare access and discrimination may be worse for those 
occupying certain marginalized social locations, including TNB individuals who are disabled, ${ }^{24}$ people of color, ${ }^{12,25}$ of lower income, ${ }^{10,19}$ middle-aged, ${ }^{26}$ self-identified as transgender (as opposed to gender nonconforming or non-binary), ${ }^{27}$ or have a history of medical transition, ${ }^{28}$ and can be worsened due to geographic location (e.g., living in the Southern or Western United States). ${ }^{19}$

Insurance-based coverage denials are a common barrier for TNB individuals in accessing medically necessary genderaffirming care. Out of all USTS respondents who sought healthcare coverage through an insurer, $25 \%$ experienced a coverage denial or other barrier to care related to being TNB in the past year. ${ }^{12}$ Comparatively, other studies have found between 11 and $24 \%$ of coverage denials among the general population. ${ }^{29}$

However, provision of gender-affirming care to TNB individuals will be limited if that care is neither affordable nor accessible. In comparison with the general population, it is more common for TNB individuals to have lower incomes, be unemployed, and lack health insurance, yet have higher educational attainment. ${ }^{12,15,30}$ While $11 \%$ of adults in the USA are uninsured, USTS respondents reported a rate of $14 \% .{ }^{12}$ One study found a greater likelihood of uninsurance among TNB people of color compared with TNB white people $(25.7 \%$ versus $17.1 \%$ ) and among TNB people compared with cisgender people in the Midwestern (20.6\% versus $13.7 \%$ ) and Southern (24.2\% versus $19.5 \%$ ) United States. ${ }^{30}$ Being uninsured is associated with substantial delays in seeking care. $^{20,31}$

Health insurance coverage does not guarantee that a TNB individual will receive gender-affirming medical care. Historically, healthcare providers could deny transgender people coverage for transition-related care by regarding transgender status as a pre-existing condition; this is no longer possible under the 2010 Patient Protection and Affordable Care Act (ACA). ${ }^{32,33}$ While Obama-era interpretations of the ACA outlawed such discrimination, a federal injunction in 2016 put this interpretation on hold, going against WPATH recommendations and leaving the legal future of non-discrimination in jeopardy. ${ }^{5,32,34}$ Further, the Trump administration has proposed rolling back gender identity-inclusive regulatory interpretations. ${ }^{34}$ As such, TNB individuals continue to face barriers and legal ambiguity regarding insurance provider decisions on which services are deemed as a "medical necessity" versus "cosmetic."1,35,36

Insurance-based non-discrimination and protection policies differ by insurance type. When the USTS was conducted during summer 2015, only the District of Columbia and a handful of state-administered Medicaid programs (e.g., California, Massachusetts) provided some level of coverage for transition-related care, while others either explicitly excluded coverage or had no overt policy. ${ }^{37}$ A greater number of states prohibited private insurance-based discrimination (e.g., Illinois, Nevada). ${ }^{37}$ As of 2014, categorical exclusions for transgender care through Medicare, a federally administered program, were overturned by the Department of Health and Human Services; as with other Medicare services, coverage decisions are now made on a case-by-case basis. ${ }^{4,38}$ Additionally, while the Veteran's Health Administration was covering comprehensive transgender-related care in $2015,{ }^{39-41}$ TRICARE through the Department of Defense had yet to issue an inclusive coverage policy. ${ }^{42}$ Finally, for further context, in 2015 , the WPATH medical necessity statement, ${ }^{5}$ as well research on the cost effectiveness of transgender-related insurance coverage, ${ }^{8}$ had yet to be released.

While non-discrimination and inclusive policies continue to be enacted today, different forms of transgender-related insurance denials continue across all insurance types. Research has neglected the association between the multiple forms of transgender-related insurance denials experienced by TNB individuals and insurance type. This study aimed to assess how type of insurance coverage (e.g., private, Medicaid) affects TNB individuals' likelihood of experiencing different forms of insurance-based denials. As insurance policy is fragmented in the USA, including by level of transgenderrelated policy development, enforcement, and coverage protections, we hypothesize that different forms of denial will differ by insurance type. Investigating this question advances our understanding of how insurance programs may contribute to the multiple and gender-specific barriers transgender people may face in accessing healthcare.

\section{METHODOLOGY}

\section{Design}

This paper utilizes secondary USTS data. As anonymized secondary data, this study received exempt approval from the University of Michigan IRB. The USTS was conducted by the National Center for Transgender Equality in 2015 to ascertain TNB experiences across multiple life experiences, including individual health status, health services access and utilization, and experiences with denials. Purposive sampling online and in partnership with over 400 organizations across the USA was used to recruit participants. Participants were eligible to complete the survey if they self-identified as TNB, were at least 18 years of age, and lived in the USA. The survey was offered in English and Spanish and was completed by 27,715 participants. Detailed methodological information can be found in the USTS report. ${ }^{12}$

As the USTS has an overrepresentation of participants who were 18 years old and White, recommended sampling weights are used. This weight was created by the USTS team to provide estimates closer to TNB population age and race attributes, based upon other studies. ${ }^{12}$

\section{Measurement}

The primary dependent variables used in this study are eight forms of self-reported insurance-based denials. These included 
when a health insurance program in the previous year had (1) not changed records to patient's current name or gender; (2) denied transition-related hormone therapy; (3) denied transition-related surgery; (4) covered only some transitionrelated surgery; (5) covered transition-related surgery, but had no in-network surgery providers; (6) denied other "genderspecific healthcare (such as Pap smears, prostate exams, mammograms)" due to the patient being transgender; and (7) denied routine healthcare due to the patient being transgender. ${ }^{12}$ These seven variables were binary $(0=$ no; $1=$ yes $)$ and included only those who accessed, or attempted to access, that form of care. Additionally, an eighth variable "any denial" was created, which collapsed observations across all seven forms of denial. If a participant experienced any one of the seven forms, they were coded as having experienced any denial. Participants were included in the final analytical sample if they were covered by an insurance type and attempted to access any of the seven forms of care $(n=11,320)$.

The primary independent variable was type of insurance. This included (1) private insurance, which included both employer-based or market-based coverage via the participant or someone else; (2) Medicare; (3) Medicaid; (4) militaryrelated, which collapsed together Veteran's Administration insurance $(n=116)$ and TRICARE $(n=133)$ due to their low sample size; and (5) multiple, for participants covered by more than one of the four insurance types. Insurance was recoded as a binary dummy variable $(0=$ does not have that type; $1=$ has type).

Other independent variables were included as controls. These included gender identity, race, educational attainment, income, census region of residence, disability, age, and history of having any transgender-related medical or surgical transition. Gender identity was a binary variable $(0=$ genderqueer/ non-binary; 1 = transgender); race, age, education, and income were categorical variables; and disability and history of transition were binary variables. The Native American $(n=134)$ and Middle Eastern $(n=44)$ race categories were dropped due to low sample size. History of transition was a USTSconstructed variable that included any transgender-related health service, including hormone therapy, surgery, and transgender-related counseling or therapy, except voice therapy.

\section{Analysis}

Descriptive results for all variables were based upon the full weighted analytic sample. Prevalence of each form of denial by type of insurance was ascertained through bivariate crosstabulation and tests of independence. Finally, multivariate logistic regressions were conducted to analyze the likelihood of experiencing all forms of denial by insurance type and the control variables. This involved examining eight models, in each of which only those participants who attempted to access the care associated with that model's form of denial (i.e., each model's dependent variable) were included. Private insurance was selected as the reference category for insurance type. Regressions were examined for variable specification and sufficient data. Acceptable levels of multicollinearity were detected, including between education and income, and between explanatory and control variables. Adjustments for multiple comparisons were deemed unnecessary as models and hypothesized associations were considered independently. ${ }^{43,44}$ Risk ratios, calculated using an adjustment method, ${ }^{45}$ and $95 \%$ confidence intervals are presented.

\section{RESULTS}

Descriptive results (Table 1) showed that $72.7 \%$ of study participants in the weighted sample were covered by private insurance $(n=8390)$, followed by $14.2 \%$ with Medicaid $(n=$ $1395)$ and $8.6 \%$ with multiple forms of insurance $(n=970)$. Most respondents self-identified as transgender $(81.1 \%)$ and White $(64.7 \%)$. Additionally, the vast majority of respondents

Table 1 Sample Characteristics $(n=11,320)$

\begin{tabular}{|c|c|c|c|}
\hline & $n$ & $\%$ & $\begin{array}{l}\text { Weighted } \\
(\%)\end{array}$ \\
\hline \multicolumn{4}{|l|}{ Insurance type } \\
\hline Private & 8390 & 74.1 & 72.7 \\
\hline Medicare & 283 & 2.5 & 2.2 \\
\hline Medicaid & 1395 & 12.3 & 14.2 \\
\hline Military-related & 282 & 2.5 & 2.3 \\
\hline Multiple & 970 & 8.6 & 8.6 \\
\hline \multicolumn{4}{|l|}{ Gender } \\
\hline Transgender & 9136 & 80.7 & 81.1 \\
\hline Genderqueer or non-binary & 2184 & 19.3 & 18.9 \\
\hline \multicolumn{4}{|l|}{ Race } \\
\hline White & 9560 & 84.5 & 64.7 \\
\hline Latino/a/Hispanic & 550 & 4.9 & 15.5 \\
\hline Black & 324 & 2.9 & 12.9 \\
\hline Asian/Native Hawaiian/Pacific & 277 & 2.5 & 4.4 \\
\hline \multicolumn{4}{|l|}{ Islander } \\
\hline Biracial/multiracial/not listed & 609 & 5.4 & 2.4 \\
\hline \multicolumn{4}{|l|}{ Education attainment } \\
\hline Less than high school & 215 & 1.9 & 1.8 \\
\hline High school & 972 & 8.6 & 8.0 \\
\hline Some college & 3685 & 32.6 & 34.5 \\
\hline Associate's degree & 1044 & 9.2 & 9.7 \\
\hline Bachelor's degree & 3381 & 29.9 & 29.0 \\
\hline Graduate or professional degree & 2023 & 17.9 & 17.1 \\
\hline \multicolumn{4}{|l|}{ Income } \\
\hline No income & 306 & 2.7 & 3.0 \\
\hline Low income $(\$ 1-\$ 10 \mathrm{k})$ & 1171 & 10.3 & 11.7 \\
\hline Low-mid income $(\$ 10 \mathrm{k}-\$ 25 \mathrm{k})$ & 2064 & 18.2 & 17.8 \\
\hline Mid income $(\$ 25 \mathrm{k}-\$ 50 \mathrm{k})$ & 2513 & 22.2 & 22.7 \\
\hline Mid-high income $(\$ 50 \mathrm{k}-\$ 100 \mathrm{k})$ & 3070 & 27.1 & 26.5 \\
\hline High income $(\$ 100 \mathrm{k}+)$ & 2196 & 19.4 & 18.4 \\
\hline \multicolumn{4}{|l|}{ Region } \\
\hline Northeast & 2472 & 21.8 & 21.3 \\
\hline Midwest & 2273 & 20.1 & 18.5 \\
\hline South & 2677 & 23.7 & 25.4 \\
\hline West & 3898 & 34.4 & 34.9 \\
\hline \multicolumn{4}{|l|}{ Age } \\
\hline $18-25$ years old & 3888 & 34.4 & 35.1 \\
\hline $26-44$ years old & 4952 & 43.8 & 45.2 \\
\hline 45-64 years old & 2131 & 18.8 & 17.2 \\
\hline $65+$ years old & 349 & 3.1 & 2.5 \\
\hline \multicolumn{4}{|l|}{ Disability } \\
\hline Yes & 2920 & 25.8 & 26.0 \\
\hline No & 8400 & 74.2 & 74.0 \\
\hline \multicolumn{4}{|l|}{ Any transition } \\
\hline Yes & 10,267 & 90.7 & 90.9 \\
\hline No & 1053 & 9.3 & 9.1 \\
\hline
\end{tabular}


Table 2 Experiences of Insurance-Based Denials: Prevalence and Relationship by Insurance Type

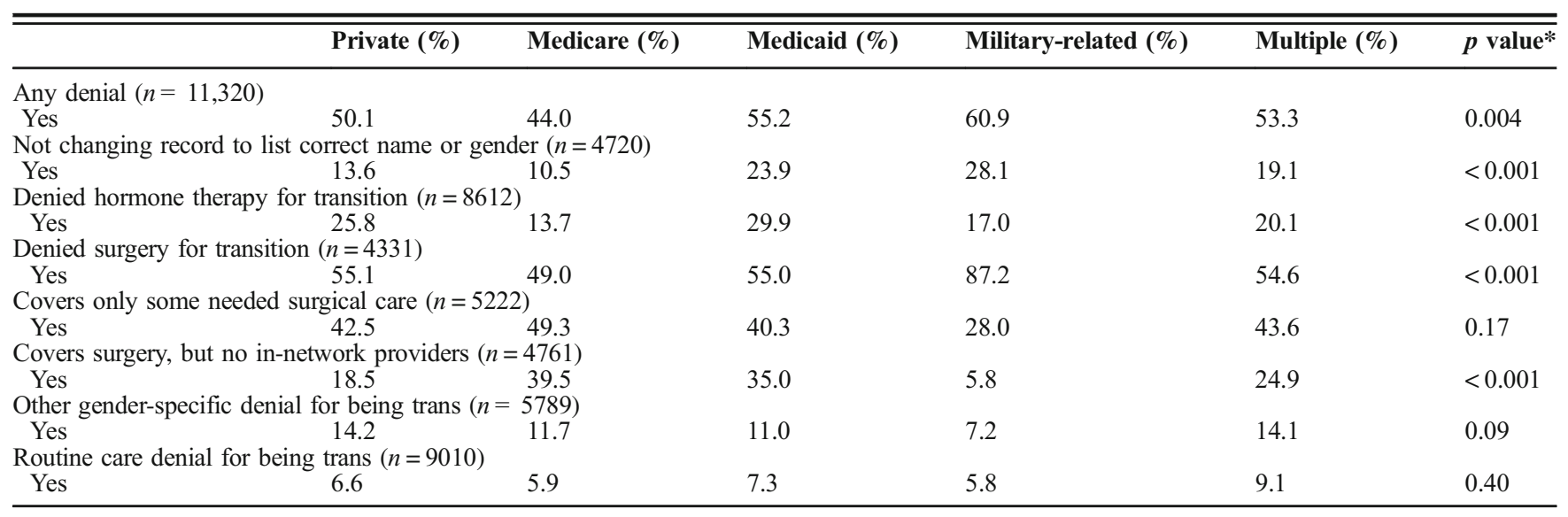

*Note: With weighted data, chi-square tests are converted into a design-based F statistic, which provides a more accurate $p$ value

had a history of transgender-related medical or surgical transition (90.9\%). Approximately one-quarter of participants were disabled (26.0\%). Most participants were 18 to 44 years old $(80.3 \%)$.

Bivariate results (Table 2) revealed a significant relationship between insurance type and five of the insurance denial variables. This included the relationship between insurance and having experienced any of the seven forms of denials ( $p=$ 0.004): people who were covered by all forms of insurance, save Medicare, were more likely to experience denials than not. This included $60.9 \%$ of those with military-related insurance and $55.2 \%$ of those with Medicaid. There are other prevalence rates of note: $55.8 \%$ of all respondents in the weighted sample who accessed surgery for transgenderrelated transition were denied insurance coverage ( $p<0.001)$, including $87.2 \%$ of those military-related insurance. The least prevalent, but still statistically significant, form of denial was not changing a record to list correct name or gender $(p<0.001) ; 15.9 \%$ of respondents experienced this form of denial, with the lowest prevalence among those with Medicare $(10.5 \%)$ and the highest among those with militaryrelated insurance $(28.1 \%)$.

Multivariate logistic regression results are presented for all eight denial variables using weighted samples (Tables 3 and 4). All eight models showed overall statistical significance (all $p<0.001$ ), and Hosmer-Lemeshow goodness of fit tests were insignificant for all models, indicating good fit and that actual and predicted frequencies were not statistically different (all $p>0.05)$. In the model in which the dependent variable is any form of denial, no form of insurance was associated with a greater likelihood of experiencing denials $(p>0.05)$.

However, when holding all other variables constant, analyses of the other models revealed several significant relationships between transgender-related denials and insurance type. All results are in comparison with the reference group of private insurance. For those who attempted to change their correct name or gender with an insurance company, those with military-related insurance were 1.93 times more likely $(\mathrm{CI}=$ $1.33-2.80 ; p=0.009)$, those with Medicaid were 1.50 times more likely $(\mathrm{CI}=1.17-1.93 ; p=0.005)$, and those with multiple forms of insurance were 1.60 times more likely $(\mathrm{CI}=1.21-$ $2.12 ; p=0.005)$ to experience this form of denial. In accessing transition-related hormone therapy, those with Medicaid were 1.22 times more likely $(\mathrm{CI}=1.05-1.42 ; p=0.02)$ to experience denials, while those with Medicare (adjusted risk ratio $(A R R)=$ $0.71 ; \mathrm{CI}=0.50-1.02 ; p=0.03)$ and military-related insurance $(\mathrm{ARR}=0.63 ; \mathrm{CI}=0.43-0.91 ; p=0.002)$ were less likely to experience these denials. However, those with military-related insurance were 1.53 times more likely to experience denials for transition-related surgery $(\mathrm{CI}=1.36-1.72 ; p<0.001)$. Those with Medicare were 1.34 times more likely $(\mathrm{CI}=1.06-1.69$; $p=0.03$ ) to have their insurance only cover some needed surgical care and 1.84 times more likely $(\mathrm{CI}=1.29-2.62 ; p=$ 0.009 ) to have their insurance cover surgery without any innetwork providers capable of offering it. Conversely, those with military-related insurance were less likely to have only some surgical coverage $(\mathrm{ARR}=0.71 ; \mathrm{CI}=0.52-0.98 ; p=0.02)$ or surgical coverage with no in-network provider $(\mathrm{ARR}=0.33$; $\mathrm{CI}=0.14-0.80 ; p<.001)$. Those with Medicaid coverage were 1.54 times more likely $(\mathrm{CI}=1.20-1.98 ; p=0.003)$ to have no in-network providers for needed surgery. Routine care denials were not significantly different across all types of insurance $(p>0.05)$.

Multivariate results also demonstrated notable significant relationships between several control variables and forms of denial. Self-identifying as transgender, in comparison with genderqueer/non-binary, was associated with a greater likelihood of experiencing denials of transition-related surgery $(\mathrm{ARR}=1.15 ; \mathrm{CI}=1.01-1.31 ; p=0.02)$, other genderspecific care $(\mathrm{ARR}=2.30 ; \mathrm{CI}=1.57-3.35 ; p<0.001)$, and routine care $(\mathrm{ARR}=1.81 ; \mathrm{CI}=1.27-2.58 ; p<0.001)$. Residing in the Midwest or South census regions was associated with an increased likelihood of experiencing many forms of denial, compared with residing in the Northeast. 
Table 3 Multivariate Adjusted Risk Ratios (ARR) of Experiences with Health Insurance Denials

\begin{tabular}{|c|c|c|c|c|c|c|c|c|}
\hline \multirow[b]{2}{*}{ Variable } & \multicolumn{2}{|c|}{ Any denial $(n=11,320)$} & \multicolumn{2}{|c|}{$\begin{array}{l}\text { Not changing record to } \\
\text { list correct name or } \\
\text { gender }(n=4720)\end{array}$} & \multicolumn{2}{|c|}{$\begin{array}{l}\text { Denied hormones for } \\
\text { transition }(n=8612)\end{array}$} & \multicolumn{2}{|c|}{$\begin{array}{l}\text { Denied surgery for } \\
\text { transition }(n=4331)\end{array}$} \\
\hline & ARR (95\% CI) & $\begin{array}{l}p \\
\text { value }\end{array}$ & ARR (95\% CI) & $\begin{array}{l}p \\
\text { value }\end{array}$ & ARR (95\% CI) & $\begin{array}{l}p \\
\text { value }\end{array}$ & $\operatorname{ARR}(95 \% \mathrm{CI})$ & $\begin{array}{l}p \\
\text { value }\end{array}$ \\
\hline \multicolumn{9}{|l|}{ Insurance } \\
\hline Private (ref) & 1.0 & & 1.0 & & 1.0 & & 1.0 & \\
\hline Medicare & $0.91(0.77-1.08)$ & 0.30 & $1.16(0.72-1.86)$ & 0.55 & $0.71(0.50-1.02)$ & 0.03 & $0.96(0.73-1.25)$ & 0.76 \\
\hline Medicaid & $1.08(0.98-1.18)$ & 0.10 & $1.50(1.17-1.93)$ & 0.005 & $1.22(1.05-1.42)$ & 0.02 & $1.04(0.92-1.17)$ & 0.52 \\
\hline Military-related & $1.13(0.99-1.30)$ & 0.07 & $1.93(1.33-2.80)$ & 0.009 & $0.63(0.43-0.91)$ & 0.002 & $1.53(1.36-1.72)$ & $\begin{array}{l}< \\
0.001\end{array}$ \\
\hline Multiple & $1.05(0.96-1.15)$ & 0.24 & $1.60(1.21-2.12)$ & 0.005 & $0.88(0.72-1.08)$ & 0.22 & $1.03(0.90-1.17)$ & 0.62 \\
\hline \multicolumn{9}{|l|}{ Gender } \\
\hline $\begin{array}{l}\text { Genderqueer/non-binary } \\
\text { (ref) }\end{array}$ & 1.0 & & 1.0 & & 1.0 & & 1.0 & \\
\hline Transgender & $1.49(1.36-1.63)$ & $\begin{array}{l}< \\
0.001\end{array}$ & $0.79(0.62-1.02)$ & 0.10 & $0.92(0.79-1.07)$ & 0.33 & $1.15(1.01-1.31)$ & 0.02 \\
\hline \multicolumn{9}{|l|}{ Race } \\
\hline White (ref) & 1.0 & & 1.0 & & 1.0 & & 1.0 & \\
\hline Latino/a/Hispanic & $1.04(0.96-1.13)$ & 0.33 & $1.26(0.98-1.62)$ & 0.10 & $1.00(0.85-1.19)$ & 0.93 & $0.93(0.83-1.05)$ & 0.25 \\
\hline Black & $1.05(0.93-1.19)$ & 0.39 & $0.98(0.69-1.40)$ & 0.95 & $0.91(0.72-1.14)$ & 0.42 & $0.68(0.57-0.82)$ & $\begin{array}{l}< \\
0.001\end{array}$ \\
\hline $\begin{array}{l}\text { Asian/Native Hawaiian/ } \\
\text { Pacific Islander }\end{array}$ & $0.85(0.74-0.98)$ & 0.02 & $1.09(0.70-1.70)$ & 0.70 & $0.83(0.63-1.09)$ & 0.16 & $0.77(0.61-0.98)$ & 0.02 \\
\hline $\begin{array}{l}\text { Biracial/multiracial/not } \\
\text { listed }\end{array}$ & $\begin{array}{l}0.99 \\
(0.91-1.08)\end{array}$ & 0.98 & $1.09(0.82-1.45)$ & 0.55 & $0.92(0.77-1.11)$ & 0.41 & $0.87(0.75-1.00)$ & 0.04 \\
\hline \multicolumn{9}{|l|}{ Education attainment } \\
\hline $\begin{array}{l}\text { Less than high school } \\
\text { (ref) }\end{array}$ & 1.0 & & 1.0 & & 1.0 & & 1.0 & \\
\hline High school & $0.99(0.78-1.24)$ & 0.94 & $0.63(0.31-1.29)$ & 0.14 & $1.15(0.75-1.75)$ & 0.54 & $0.96(0.68-1.37)$ & 0.86 \\
\hline Some college & $1.04(0.84-1.29)$ & 0.67 & $0.66(0.36-1.19)$ & 0.16 & $1.17(0.77-1.77)$ & 0.45 & $1.03(0.75-1.41)$ & 0.85 \\
\hline Associate's degree & $1.06(0.86-1.31)$ & 0.56 & $0.63(0.32-1.25)$ & 0.13 & $1.29(0.87-1.93)$ & 0.25 & $1.03(0.75-1.43)$ & 0.82 \\
\hline Bachelor's degree & $1.04(0.84-1.29)$ & 0.68 & $0.57(0.30-1.07)$ & 0.06 & $1.19(0.79-1.78)$ & 0.42 & $0.99(0.77-1.37)$ & 0.98 \\
\hline $\begin{array}{l}\text { Graduate or professional } \\
\text { degree }\end{array}$ & $\begin{array}{l}1.10 \\
(0.89-1.35)\end{array}$ & 0.36 & $0.67(0.34-1.30)$ & 0.19 & $1.26(0.85-1.88)$ & 0.28 & $0.97(0.69-1.35)$ & 0.88 \\
\hline \multicolumn{9}{|l|}{ Income } \\
\hline No income (ref) & 1.0 & & 1.0 & & 1.0 & & 1.0 & \\
\hline Low income (\$1-\$10k) & $\begin{array}{l}1.26 \\
(1.09-1.46)\end{array}$ & 0.004 & $2.18(1.27-3.73)$ & 0.04 & $1.25(0.90-1.72)$ & 0.21 & $1.17(0.94-1.46)$ & 0.18 \\
\hline $\begin{array}{l}\text { Low-mid income } \\
(\$ 10 \mathrm{k}-\$ 25 \mathrm{k})\end{array}$ & $1.25(1.09-1.43)$ & 0.003 & $2.30(1.35-3.92)$ & 0.02 & $1.34(0.98-1.83)$ & 0.09 & $1.21(0.98-1.50)$ & 0.08 \\
\hline $\begin{array}{l}\text { Mid income } \\
(\$ 25 \mathrm{k}-\$ 50 \mathrm{k})\end{array}$ & $1.26(1.09-1.45)$ & 0.002 & $2.31(1.38-3.87)$ & 0.02 & $1.34(0.98-1.84)$ & 0.09 & $1.24(1.00-1.53)$ & 0.06 \\
\hline $\begin{array}{l}\text { Mid-high income } \\
(\$ 50 \mathrm{k}-\$ 100 \mathrm{k})\end{array}$ & $1.25(1.08-1.44)$ & 0.003 & $1.83(1.05-3.18)$ & 0.08 & $1.27(0.93-1.74)$ & 0.15 & $1.21(0.97-1.51)$ & 0.09 \\
\hline High income $(\$ 100 \mathrm{k}+)$ & $1.17(1.01-1.36)$ & 0.04 & $1.66(0.93-2.97)$ & 0.16 & $1.16(0.83-1.61)$ & 0.39 & $1.11(0.88-1.39)$ & 0.38 \\
\hline \multicolumn{9}{|l|}{ Region } \\
\hline Northeast (ref) & 1.0 & & 1.0 & & 1.0 & & 1.0 & \\
\hline Midwest & $1.11(1.04-1.19)$ & 0.001 & $1.29(1.00-1.66)$ & 0.07 & $1.27(1.12-1.46)$ & 0.001 & $1.40(1.29-1.51)$ & $\begin{array}{l}< \\
0.001\end{array}$ \\
\hline South & $1.11(1.04-1.19)$ & 0.001 & $1.49(1.16-1.91)$ & 0.005 & $1.41(1.24-1.62)$ & $\begin{array}{l}< \\
0.001\end{array}$ & $1.33(1.22-1.45)$ & $\begin{array}{l}< \\
0.001\end{array}$ \\
\hline West & $1.06(0.99-1.14)$ & 0.07 & $1.02(0.80-1.29)$ & 0.86 & $0.87(0.75-1.01)$ & 0.07 & $0.92(0.84-1.01)$ & 0.09 \\
\hline \multicolumn{9}{|l|}{ Age } \\
\hline $65+$ years old (ref) & 1.0 & & 1.0 & & 1.0 & & 1.0 & \\
\hline $18-25$ years old & $1.49(1.33-1.67)$ & $\begin{array}{l}< \\
0.001\end{array}$ & $3.90(2.68-5.67)$ & $\begin{array}{l}< \\
0.001\end{array}$ & $1.87(1.37-2.55)$ & 0.001 & $1.22(1.00-1.48)$ & 0.046 \\
\hline 26-44 years old & $1.43(1.26-1.63)$ & $\begin{array}{l}< \\
0.001\end{array}$ & $3.48(2.34-5.18)$ & $\begin{array}{l}< \\
0.001\end{array}$ & $1.53(1.12-2.10)$ & 0.01 & $1.23(1.01-1.50)$ & 0.03 \\
\hline 45-64 years old & $1.32(1.19-1.48)$ & $\begin{array}{l}< \\
0.001\end{array}$ & $2.36(1.52-3.66)$ & 0.006 & $1.01(0.72-1.43)$ & 0.92 & $1.09(0.91-1.32)$ & 0.34 \\
\hline Disability & $1.13(1.07-1.20)$ & $<0.001$ & $1.27(1.03-1.56)$ & 0.03 & $1.13(1.00-1.27)$ & 0.04 & $1.05(0.96-1.14)$ & 0.24 \\
\hline Any transition & $1.39(1.15-1.67)$ & $<0.001$ & $0.68(0.46-1.00)$ & 0.10 & $0.64(0.50-0.82)$ & 0.004 & $0.94(0.78-1.14)$ & 0.60 \\
\hline
\end{tabular}


Table 4 Multivariate Adjusted Risk Ratios (ARR) of Experiences with Health Insurance Denials

\begin{tabular}{|c|c|c|c|c|c|c|c|c|}
\hline \multirow[b]{2}{*}{ Variable } & \multicolumn{2}{|c|}{$\begin{array}{l}\text { Covers only some needed } \\
\text { surgical care }(n=5222)\end{array}$} & \multicolumn{2}{|c|}{$\begin{array}{l}\text { Covers surgery, but no } \\
\text { in-network providers } \\
(n=4761)\end{array}$} & \multicolumn{2}{|c|}{$\begin{array}{l}\text { Other gender-specific } \\
\text { denial for being trans } \\
(n=5789)\end{array}$} & \multicolumn{2}{|c|}{$\begin{array}{l}\text { Routine care denial for } \\
\text { being trans }(n=9010)\end{array}$} \\
\hline & ARR (95\% CI) & $\begin{array}{l}p \\
\text { value }\end{array}$ & $\operatorname{ARR}(95 \% \mathrm{CI})$ & $\begin{array}{l}p \\
\text { value }\end{array}$ & ARR (95\% CI) & $\begin{array}{l}p \\
\text { value }\end{array}$ & $\operatorname{ARR}(95 \% \mathrm{CI})$ & $\begin{array}{l}p \\
\text { value }\end{array}$ \\
\hline \multicolumn{9}{|l|}{ Insurance } \\
\hline Private (ref) & 1.0 & & 1.0 & & 1.0 & & 1.0 & \\
\hline Medicare & $1.34(1.06-1.69)$ & 0.03 & $1.84(1.29-2.62)$ & 0.009 & $0.79(0.49-1.28)$ & 0.30 & $0.95(0.54-1.68)$ & 0.87 \\
\hline Medicaid & $1.01(0.84-1.22)$ & 0.87 & $1.54(1.20-1.98)$ & 0.003 & $0.73(0.51-1.03)$ & 0.049 & $0.90(0.63-1.29)$ & 0.57 \\
\hline Military-related & $0.71(0.52-0.98)$ & 0.02 & $0.33(0.14-0.80)$ & $\begin{array}{l}< \\
0.001\end{array}$ & $0.42(0.23-0.74)$ & $\begin{array}{l}< \\
0.001\end{array}$ & $0.74(0.31-1.75)$ & 0.45 \\
\hline Multiple & $1.16(0.99-1.35)$ & 0.07 & $1.18(0.89-1.55)$ & 0.26 & $0.91(0.64-1.30)$ & 0.61 & $1.30(0.87-1.92)$ & 0.24 \\
\hline Gender & & & & & & & & \\
\hline $\begin{array}{l}\text { Genderqueer/non-binary } \\
\text { (ref) }\end{array}$ & 1.0 & & 1.0 & & 1.0 & & 1.0 & \\
\hline Transgender & $1.08(0.92-1.26)$ & 0.32 & $0.94(0.73-1.20)$ & 0.65 & $2.30(1.57-3.35)$ & $\begin{array}{l}< \\
0.001\end{array}$ & $1.81(1.27-2.58)$ & $\begin{array}{l}< \\
0.001\end{array}$ \\
\hline \multicolumn{9}{|l|}{ Race } \\
\hline White (ref) & 1.0 & & 1.0 & & 1.0 & & 1.0 & \\
\hline Latino/a/Hispanic & $0.93(0.80-1.08)$ & 0.37 & $0.97(0.76-1.25)$ & 0.87 & $1.10(0.83-1.44)$ & 0.51 & $1.42(1.03-1.95)$ & 0.06 \\
\hline Black & $0.83(0.65-1.05)$ & 0.10 & $1.16(0.86-1.56)$ & 0.35 & $0.84(0.58-1.22)$ & 0.34 & $1.24(0.84-1.84)$ & 0.32 \\
\hline $\begin{array}{l}\text { Asian/Native Hawaiian/ } \\
\text { Pacific Islander }\end{array}$ & $0.97(0.78-1.21)$ & 0.82 & $1.22(0.89-1.69)$ & 0.25 & $0.62(0.35-1.10)$ & 0.04 & $0.98(0.55-1.74)$ & 0.95 \\
\hline $\begin{array}{l}\text { Biracial/multiracial/not } \\
\text { listed }\end{array}$ & $0.96(0.82-1.13)$ & 0.68 & $1.31(1.05-1.63)$ & 0.03 & $0.90(0.66-1.23)$ & 0.51 & $1.04(0.73-1.49)$ & 0.81 \\
\hline \multicolumn{9}{|l|}{ Education attainment } \\
\hline $\begin{array}{l}\text { Less than high school } \\
\text { (ref) }\end{array}$ & 1.0 & & 1.0 & & 1.0 & & 1.0 & \\
\hline High school & $0.84(0.54-1.28)$ & 0.39 & $0.51(0.26-1.00)$ & 0.01 & $0.79(0.31-1.98)$ & 0.58 & $0.58(0.24-1.42)$ & 0.15 \\
\hline Some college & $0.84(0.57-1.23)$ & 0.36 & $0.77(0.44-1.34)$ & 0.35 & $1.24(0.54-2.85)$ & 0.62 & $0.60(0.28-1.29)$ & 0.18 \\
\hline Associate's degree & $0.75(0.48-1.16)$ & 0.15 & $0.73(0.39-1.35)$ & 0.26 & $1.24(0.54-2.85)$ & 0.63 & $0.76(0.33-1.73)$ & 0.49 \\
\hline Bachelor's degree & $0.89(0.61-1.30)$ & 0.55 & $0.71(0.40-1.26)$ & 0.22 & $1.24(0.54-2.79)$ & 0.62 & $0.63(0.29-1.36)$ & 0.22 \\
\hline $\begin{array}{l}\text { Graduate or professional } \\
\text { degree }\end{array}$ & $0.96(0.66-1.41)$ & 0.86 & $0.85(0.47-1.51)$ & 0.57 & $1.38(0.61-3.10)$ & 0.48 & $0.62(0.28-1.40)$ & 0.21 \\
\hline \multicolumn{9}{|l|}{ Income } \\
\hline No income (ref) & 1.0 & & 1.0 & & 1.0 & & 1.0 & \\
\hline Low income $(\$ 1-\$ 10 \mathrm{k})$ & $1.09(0.77-1.55)$ & 0.62 & $1.11(0.66-1.86)$ & 0.70 & $0.92(0.49-1.71)$ & 0.80 & $1.27(0.64-2.50)$ & 0.53 \\
\hline $\begin{array}{l}\text { Low-mid income } \\
(\$ 10 \mathrm{k}-\$ 25 \mathrm{k})\end{array}$ & $1.07(0.78-1.47)$ & 0.65 & $0.72(0.43-1.18)$ & 0.15 & $0.92(0.51-1.66)$ & 0.80 & $0.96(0.49-1.88)$ & 0.91 \\
\hline $\begin{array}{l}\text { Mid income } \\
(\$ 25 \mathrm{k}-\$ 50 \mathrm{k})\end{array}$ & $1.21(0.90-1.63)$ & 0.22 & $0.85(0.53-1.37)$ & 0.51 & $0.80(0.44-1.46)$ & 0.45 & $1.02(0.53-1.99)$ & 0.93 \\
\hline $\begin{array}{l}\text { Mid-high income } \\
(\$ 50 \mathrm{k}-\$ 100 \mathrm{k})\end{array}$ & $1.13(0.83-1.54)$ & 0.42 & $0.88(0.55-1.41)$ & 0.59 & $0.75(0.41-1.39)$ & 0.35 & $0.80(0.41-1.58)$ & 0.52 \\
\hline High Income $(\$ 100 \mathrm{k}+)$ & $1.23(0.92-1.64)$ & 0.19 & $1.00(0.63-1.59)$ & 0.97 & $0.83(0.44-1.54)$ & 0.54 & $1.07(0.54-2.10)$ & 0.84 \\
\hline \multicolumn{9}{|l|}{ Region } \\
\hline Northeast (ref) & 1.0 & & 1.0 & & 1.0 & & 1.0 & \\
\hline Midwest & $0.85(0.74-0.98)$ & 0.02 & $0.55(0.42-0.71)$ & $\begin{array}{l}< \\
0.001\end{array}$ & $1.07(0.84-1.38)$ & 0.56 & $1.55(1.11-2.15)$ & 0.02 \\
\hline South & $0.88(0.77-1.01)$ & 0.06 & $0.67(0.53-0.85)$ & $\begin{array}{l}< \\
0.001\end{array}$ & $1.28(1.00-1.64)$ & 0.06 & $1.67(1.21-2.30)$ & 0.01 \\
\hline West & $1.10(0.97-1.24)$ & 0.13 & $1.09(0.90-1.32)$ & 0.38 & $0.89(0.70-1.13)$ & 0.35 & $1.18(0.85-1.62)$ & 0.33 \\
\hline \multicolumn{9}{|l|}{ Age } \\
\hline $65+$ years old (ref) & 1.0 & & 1.0 & & 1.0 & & 1.0 & \\
\hline $18-25$ years old & $1.65(1.33-2.07)$ & $\begin{array}{l}< \\
0.001\end{array}$ & $1.03(0.65-1.63)$ & 0.88 & $1.13(0.68-1.89)$ & 0.64 & $1.96(1.03-3.74)$ & 0.10 \\
\hline 26-44 years old & $1.47(1.15-1.88)$ & 0.002 & $0.83(0.53-1.29)$ & 0.42 & $1.54(0.96-2.47)$ & 0.08 & $2.66(1.45-4.84)$ & 0.01 \\
\hline 45-64 years old & $1.34(1.07-1.68)$ & 0.02 & $1.01(0.66-1.55)$ & 0.95 & $0.96(0.59-1.55)$ & 0.87 & $1.93(1.06-3.53)$ & 0.10 \\
\hline Disability & $1.06(0.94-1.19)$ & 0.33 & $1.41(1.18-1.69)$ & 0.001 & $1.26(1.02-1.56)$ & 0.04 & $1.31(1.01-1.70)$ & 0.05 \\
\hline Any transition & $1.10(0.78-1.55)$ & 0.53 & $0.86(0.56-1.34)$ & 0.56 & $1.90(0.97-3.73)$ & 0.01 & $1.22(0.67-2.20)$ & 0.47 \\
\hline
\end{tabular}

\section{DISCUSSION}

This study is the first to examine the association between multiple forms of TNB insurance-based denial and type of insurance. Consistent with other studies that document barriers for TNB individuals in accessing healthcare services, ${ }^{10,17-22,}$ 24-28 we find that transgender-related insurance denials are pervasive. Further, these findings augment other discussions of trans-related policy issues by detailing the effects of insurance policy on specific healthcare needs. ${ }^{4,8,32-36}$
These results help direct TNB health insurance policy discussions. For instance, results show that private insurance may be less prone to denials than other forms of insurance across many health needs, which may reflect the large number of state-based anti-discrimination protections for private insurance, as well as their effectiveness. ${ }^{37}$ However, this may not be relevant for employer selfinsured plans, which are not affected by state-based protections. ${ }^{46}$ Instead, since private insurance is the most 
common type covering the TNB population, lower likelihoods of denial may be due to private insurance companies benefitting from both more experience in serving TNB people and private employers wanting to retain employees. Further, there is an association between Medicare coverage and only having some medically necessary surgeries covered; this is coherent with current Medicare policy that relegates coverage decisions to a local, caseby-case - and thus inconsistent-basis. ${ }^{32,38,47}$ Medicaidbased denials regarding hormone denials and name or gender changes may reflect the large number of states that excludes or has no policy governing gender-affirming medical care, ${ }^{37}$ or the varying state-level definitions of medical necessity. ${ }^{48}$ A lack of in-network providers for surgery may reflect the need for insurers to purposely incorporate qualified TNB-affirming providers into their networks. Both explicit non-discrimination policies and inclusion of gender-affirming medical providers in network are needed to advance TNB health. Finally, military-related insurance denials are of particular concern, as trans veterans already face high disparities in physical and mental health (e.g., depression) compared with cisgender veterans, which may be exacerbated by trans-specific care denials. ${ }^{39}$ However, further research that can tease apart TRICARE versus VA insurance experiences is needed.

Findings are also consistent with other research in showing that geographic region and gender identity as important factors in TNB denials. ${ }^{19,27}$ First, barriers to TNB care are greater for those living in the Southern United States. However, our analysis also shows similarly increased likelihood of denials in the Midwest. This is consistent with state-based policy tracking that shows that in 2015, nearly no state in the Midwest or South had non-discrimination policy for private insurance or Medicaid. ${ }^{37}$ Second, other research has similarly shown that self-identifying as transgender, as compared with non-binary, is associated with greater medical care refusals due to bias. ${ }^{27}$ This may be related to how much transition-related medical care TNB individuals have received previously, ${ }^{28}$ but more research is needed to understand this comparison.

\section{Limitations}

Several limitations are of note in this study. First, it used crosssectional data that only captured 12 months of singular retrospective experiences with insurance denials. Longitudinal data is needed to capture if denials are pervasive over time as care needs persist. Relatedly, the USTS provides service user experiences, but no data on the insurance provider end. Due to this, we are limited in fully attributing to insurance companies a denial like changing gender on insurance records since participants with employer-based private insurance may have actually experienced this denial from their employer during enrollment; however, preliminary analysis showed no difference in this form of denial between employer-based and individually purchased insurance. Evidence like insurance claims data could provide information on systematic trends and insurer reasons for denial. ${ }^{49}$ Finally, as each regression model is based on different, if somewhat overlapping, subsamples, readers are cautioned regarding making simple comparisons between models. ${ }^{50}$

Subsequent research, including up-to-date self-report surveys, should update these findings with policy today ${ }^{37}$ and track them over time, as insurance policies are affected by greater TNB inclusion, expansive coverage, and nondiscrimination laws. This will help us understand if de jure anti-discrimination laws are truly protective against unjust insurance-based denials and if other reasons for denials, such as lack of coverage eligibility, are occurring instead. Research should also evaluate if insurance non-discrimination policy has an impact on promoting non-discrimination in other areas, ${ }^{34}$ such as the direct provider level. Additionally, other transgender-related experiences, such as differences by sex assigned at birth, should be explored.

\section{CONCLUSION}

This study investigated how type of insurance coverage affects TNB individuals' experiences with different forms of insurance-based denials. This contributes to both research on trans-specific healthcare and insurance-related policy discussions by showing that insurance type is a significant predictor of multiple, specific, and prevalent forms of denials among the TNB population. These results provide continuing support for broad non-discrimination policy efforts, while also directing our attention to targeted insurance policy interventions by form of denial, which can promote equitable access for TNB people across all healthcare needs.

Acknowledgments: The authors would like to thank the United States Transgender Study research team for the access to survey data, as well as Dr. Ashley Lacombe-Duncan for her helpful comments.

Corresponding Author: Matthew Bakko, MSW, MA; School of Social Work, Department of Sociology, University of Michigan, Ann Arbor, MI, USA (e-mail: mbakko@umich.edu).

\section{Compliance with Ethical Standards:}

As anonymized secondary data, this study received exempt approval from the University of Michigan IRB.

Conflict of Interest: No conflict of interest exists for any of the authors.

\section{REFERENCES}

1. Conard LAE, Corathers SD, Trotman G. Caring for transgender and gender-nonconforming youth. Curr Pediatr Rep 2018;6(2):139-146. 
2. Davidson M. Seeking refuge under the umbrella: Inclusion, exclusion, and organizing within the category transgender. Sex Res Soc Policy 2007;4(4):60-80.

3. Downing JM, Przedworski JM. Health of Transgender Adults in the US, 2014-2016. Am J Prev Med 2018;55(3):336-344.

4. Padula WV, Baker K. Coverage for gender-affirming care: Making health insurance work for transgender Americans. LGBT Health 2017;4(4):244247.

5. World Professional Association for Transgender Health (WPATH). Position statement on medical necessity of treatment, sex reassignment, and insurance coverage in the USA; 2016

6. De Vries AL, McGuire JK, Steensma TD, et al. Young adult psychological outcome after puberty suppression and gender reassignment. Pediatrics. 2014;134(4):696-704.

7. Sevelius JM. Gender affirmation: A framework for conceptualizing risk behavior among transgender women of color. Sex Roles 2013;68(1112):675-689.

8. Padula WV, Heru S, Campbell JD. Societal implications of health insurance coverage for medically necessary services in the U.S. transgender population: A cost-effectiveness analysis. J Gen Intern Med 2016;31(4):394-401.

9. Fredriksen-Goldsen KI, Kim HJ, Emlet CA, et al. The aging and health report: Disparities and resilience among lesbian, gay, bisexual, and transgender older adults. 2011. Seattle: Institute for Multigenerational Health.

10. Grant $\mathbf{J}$, Mottet $\mathbf{L}$, Tanis $\mathbf{J}$, et al. Injustice at every turn: A report of the national transgender discrimination survey. 2011. Washington, DC, National Center for Transgender Equality and National Gay and Lesbian Task Force.

11. Gonzales G, Henning-Smith C. Barriers to care among transgender and gender nonconforming adults. Milbank Q 2017;95(4):726-748.

12. James SE, Herman JL, Rankin S, et al. The report of the 2015 U.S. transgender survey. 2016. Washington, DC, National Center for Transgender Equality.

13. Rider GN, McMorris BJ, Gower AL, et al. Health and care utilization of transgender and gender nonconforming youth: A population-based study. Pediatrics. 2018;141(3):e20171683.

14. Services and Advocacy for GLBT Elders (SAGE), National Center for Transgender Equality (NCTE), Auldridge A, et al. Improving the lives of transgender adults. New York and Washington, DC, SAGE and NCTE; 2012 .

15. Streed CG, McCarthy EP, Haas JS. Association between gender minority status and self-reported physical and mental health in the United States. JAMA Intern Med 2017;177(8):1210-1212.

16. Veale JF, Watson RJ, Peter T, Saewyc EM. The mental health of Canadian transgender youth compared with the Canadian population. J Adolesc Health 2017;60(1):44-49.

17. Gordon AR, Krieger N, Okechukwu CA, et al. Decrements in healthrelated quality of life associated with gender nonconformity among US adolescents and young adults. Qual Life Res 2017;26(8):2129-2138.

18. Mizock L, Mueser KT. Employment, mental health, internalized stigma, and coping with transphobia among transgender individuals. Psychol Sex Orientat Gend Divers 2014;1(2):146-158.

19. White Hughto JM, Murchison GR, Clark K, et al. Geographic and individual differences in healthcare access for US transgender adults: A multilevel analysis. LGBT Health 2016;3(6):424-433.

20. White Hughto JM, Reisner SL, Pachankis JE. Transgender stigma and health: A critical review of stigma determinants, mechanisms, and interventions. Soc Sci Med 2015; 147:222-231.

21. Kenagy GP, Bostwick WB. Health and social service needs of transgender people in Chicago. Int J Transgend 2005;8(2-3):57-66.

22. Lerner JE, Robles G. Perceived barriers and facilitators to health care utilization in the United States for transgender people: A review of recent literature. J Health Care Poor Underserved 2017;28(1):127-152.

23. Stotzer RL, Silverschanz $\mathbf{P}$, Wilson A. Gender identity and social services: Barriers to care. J Soc Serv Res 2013;39(1):63-77.

24. Kattari SK, Walls NE, Speer SR. Differences in experiences of discrimination in accessing social services among transgender/gender nonconforming individuals by (Dis) ability. J Soc Work Disabil Rehabil 2017;16(2):116-140.

25. Kattari SK, Walls NE, Whitfield DL, Langenderfer-Magruder L. Racial and ethnic differences in experiences of discrimination in accessing health services among transgender people in the United States. Int $\mathrm{J}$ Transgend 2015;16(2):68-79.
26. Kattari SK, Hasche $\mathbf{L}$. Differences across age groups in transgender and gender non-conforming people's experiences of health care discrimination, harassment, and victimization. J Aging Health 2016;28(2):285-306.

27. Harrison J, Grant J, Herman, JL. A gender not listed here: Genderqueers, gender rebels, and otherwise in the National Transgender Discrimination Survey. LGBTQ Public Policy J Harv Kennedy Sch 2012;2(1): 13 .

28. Miller LR, Grollman, EA. The social costs of gender nonconformity for transgender adults: Implications for discrimination and health. Sociol Forum 2015;30(3):809-831.

29. Doctor-Patient Rights Project. Not what the doctor ordered: Barriers to healthcare access for patients. Washington, DC; 2017

30. Dickey LM, Budge SL, Katz-Wise SL, Garza MV. Health disparities in the transgender community: Exploring differences in insurance coverage. Psychol Sex Orientat Gend Divers 2016;3(3):275-282.

31. Cruz TM. Assessing access to care for transgender and gender nonconforming people: A consideration of diversity in combating discrimination. Soc Sci Med 2014;110:65-73.

32. Baker KE. The future of transgender coverage. N Engl $\mathrm{J}$ Med 2017;376(19):1801-1804.

33. Stroumsa D. The state of transgender health care: policy, law, and medical frameworks. Am J Public Health Res 2014;104(3):e31-e38.

34. Underhill K. Raising the Stakes for Nondiscrimination Protections in the ACA. Hast Cent Rep 2018;48(1):8-9.

35. Khan, L. Transgender health at the crossroads: Legal norms, insurance markets, and the threat of healthcare reform. Yale $\mathrm{J}$ Health Policy Law Ethics 2011;11:375-418.

36. Rosh S. Beyond categorical exclusions: Access to transgender healthcare in state Medicaid programs. Columbia J Law Soc Probl 2017;51:1-37.

37. Movement Advancement Project (MAP). Healthcare laws and policies; 2019 [cited 2019 April 15th]; Available from http://www.lgbtmap.org/ equality-maps/healthcare_laws_and_policies.

38. National Center for Transgender Equality (NCTE). Medicare and Transgender People; 2014 [cited 2019 April 15th]; Available from https:// transequality.org/sites/default/files / docs / kyr / MedicareAndTransPeople.pdf.

39. Brown GR, Jones KT. Mental health and medical health disparities in 5135 transgender veterans receiving healthcare in the Veterans Health Administration: A case-control study. LGBT Health 2016;3(2):122-131.

40. Department of Veterans Affairs, Veterans Health Administration. VHA Directive 2013-003: Providing health care for transgender and intersex veterans; 2013 [cited 2019 April 15th]; Available from www. va.gov/ vhapublications/ViewPublication.asp?pub_ID=2863.

41. National Center for Transgender Equality (NCTE). Veterans Health Administrations Transgender Healthcare Directive; 2011 [cited 2019 April 15th]; Available from https://transequality.org/sites/ default/ files/docs/resources/VHA_Trans_Health.pdf.

42. Bushatz A. Defense Department Releases New Tricare Transgender Policy; 2016 [cited 2019 April 15th]; Available from https://www. military.com/daily-news/2016/09/06/defense-department-releasesnew-tricare-transgender-policy.html.

43. Rothman KJ. No adjustments are needed for multiple comparisons. Epidemiology. 1990;1(1):43-46.

44. Perneger TV. What's wrong with Bonferroni adjustments. BMJ. 1998;316(7139): 1236-1238.

45. Norton EC, Miller MM, Kleinman LC. Computing adjusted risk ratios and risk differences in Stata. Stata J 2013;13(3):492-509.

46. Center of Excellence for Transgender Health, Wilson AA, Green J. Health insurance coverage issues for transgender people in the United States. 2016. Available from https://transcare.ucsf.edu/ guidelines/insurance.

47. Centers for Medicare and Medicaid Services. Decision memo for gender dysphoria and gender reassignment surgery (CAG-00446N); 2016 [cited 2019 April 15th]; Available from https://www.cms.gov/medicare-coverage-database/details/nca-decision-memo.aspx?NCAId=282.

48. National Academy for State Health Policy. Medical Necessity; 2013 [cited 2019 April 15th]; Available from https://nashp.org/medical-necessity.

49. Proctor $\mathbf{K}$, Haffer SC, Ewald $\mathbf{E}$, et al. Identifying the transgender population in the Medicare Program. Transgend Health 2016;1(1):250265.

50. Norton EC, Dowd BE, Maciejewski ML. Odds ratios-current best practice and use. JAMA. 2018;320(1):84-85.

Publisher's Note Springer Nature remains neutral with regard to jurisdictional claims in published maps and institutional affiliations. 